\title{
Thermal and Mechanical Cracking in Bis(triisopropylsilylethnyl) Pentacene Thin Films
}

\author{
JHUA CHEN, ${ }^{1}$ CHEE KEONG TEE, ${ }^{2}$ JUNYAN YANG, ${ }^{3,{ }^{*}}$ CHARLES SHAW, ${ }^{1}$ MAX SHTEIN, ${ }^{3}$ \\ JOHN ANTHONY, ${ }^{5}$ DAVID C. MARTIN ${ }^{1,3,4}$ \\ ${ }^{1}$ Macromolecular Science and Engineering Center, University of Michigan, Ann Arbor, Michigan 48109 \\ ${ }^{2}$ Department of Electrical Engineering and Computer Science, University of Michigan, Ann Arbor, Michigan 48109 \\ ${ }^{3}$ Department of Materials Science and Engineering, University of Michigan, Ann Arbor, Michigan 48109 \\ ${ }^{4}$ Department of Biomedical Engineering, University of Michigan, Ann Arbor, Michigan 48109 \\ ${ }^{5}$ Department of Chemistry, University of Kentucky, Lexington, Kentucky 40506
}

Received 6 June 2006; revised 30 August 2006; accepted 4 September 2006

DOI: 10.1002 / polb.21007

Published online in Wiley InterScience (www.interscience.wiley.com).

\begin{abstract}
Bis(triisopropylsilylethnyl) pentacene (TIPS pentacene) was synthesized to increase its solubility in common liquid solvents and, at the same time, enhance the $\pi-\pi$ stacking between neighboring acenes in the crystallized state in comparison with unmodified pentacene. Hot-stage microscopy experiments revealed that during heating voids develop along the long axis of the TIPS pentacene films falong the [210] direction/parallel to the (120) planes\} and crystals overlap along the short axis \{along the [120] direction/parallel to the (210) planes\}. From molecular mechanics simulations, the predominant twin boundaries of $(\overline{120})$ and commonly observed cracking planes of (120), $(\overline{120})$, and (210) had relatively low surface energies in comparison with planes with similar Miller indices. Organic thin-film transistors with TIPS pentacene as the active layer were fabricated, and the mobility values decreased from $0.4-1.0 \mathrm{~cm}^{2} / \mathrm{V}$ s before cracking to $\sim 0.2 \mathrm{~cm}^{2} / \mathrm{V}$ s after cracking. To maintain the high charge carrier mobility of TIPS pentacene devices, these cracks should be avoided. C 2006 Wiley Periodicals, Inc. J Polym Sci Part B: Polym Phys 44: 3631-3641, 2006

Keywords: annealing; charge transport; functionalized pentacene; organic semiconductors; organic thin-film transistors; structure-property relations; surface energy
\end{abstract}

\section{INTRODUCTION}

Pentacene is an organic semiconductor with one of the highest measured hole mobilities in comparison with other similar materials, such as

This article includes Supplementary Material available from the authors upon request or via the Internet at www. interscience.wiley.com/jpages/0887-6266/suppmat

*Present address: Dow Chemical Company, Freeport, Texas.

Correspondence to: D. C. Martin (E-mail: milty@umich.edu)

Journal of Polymer Science: Part B: Polymer Physics, Vol. 44, 3631-3641 (2006) @ 2006 Wiley Periodicals, Inc. polythiophene, oligothiophenes, and phthalocyanines. However, the experimentally observed mobilities in thin films have not increased significantly since devices with $\sim 1.5 \mathrm{~cm}^{2} / \mathrm{V}$ s were reported. ${ }^{1,2}$ To improve its solid-state stacking and solubility in organic solvents, bis(triisopropylsilylethnyl) pentacene (TIPS pentacene) has been designed and synthesized with the addition of two side groups. ${ }^{3}$ The herringbone crystal structure of pentacene polymorphs is disrupted by the bulky side groups, and the TIPS pentacene molecules assume two-dimensional $\pi-\pi$ 
stacking within the unit cell. At the same time, this functionalized pentacene can be readily processed from common organic solvents such as chloroform, tetrahydrofuran, toluene, and bromobenzene. ${ }^{4}$

In this study, crack propagation was examined in TIPS pentacene crystals and crystalline thin films. Crack propagation along the (120), (120), and (210) planes was identified upon heating or compressive deformation, and a phase transition at $124{ }^{\circ} \mathrm{C}$ was associated with the observed thermal cracking. Organic thin-film transistors with TIPS pentacene as the active layer were fabricated in a controlled solution process chamber, and the mobilities of the TIPS pentacene thin-film transistors dropped by 50 $80 \%$ after thermal cracking. In addition, the surface energies of different crystallographic planes were simulated with the Cerius ${ }^{2}$ molecular simulation platform. According to the simulations, twin boundaries and observed cracking planes in TIPS pentacene had lower surface energy than planes with similar Miller indices. The calculated surface energies were similar for different planes, and this could account for the fact that different cracking directions existed in the TIPS pentacene films. The total energy of the TIPS pentacene crystals was found to go through several local maxima and minima during an increase in the separation distance in the surface energy simulations.

\section{EXPERIMENTAL}

Temperature-controlled optical microscopy was conducted on a Linkam TH 1500 hot stage, a Spot RT Color 2.2.1 CCD camera (Diagnostic Instruments, Inc.), and a Nikon OptiPhot2-POL scope. The heating and cooling rates were $10{ }^{\circ} \mathrm{C} / \mathrm{min}$, and the samples were held at the higher temperature limits for $5 \mathrm{~min}$. TIPS pentacene powders were used for variable-temperature X-ray diffraction (XRD) scans with a Bruker AXS D8 Advance powder XRD system. For each temperature, the samples were scanned for about $24 \mathrm{~min}$ at a scanning rate of $2 \% \mathrm{~min}$ and with a $2 \theta$ angle scanning range of $3-50^{\circ}$. The heating and cooling rates between the temperature scans were approximately $6{ }^{\circ} \mathrm{C} /$ min. To interpret the results from hot-stage XRD and optical microscopy, molecular simulations with Cerius $^{2}$ modeling software from Accelrys were used for comparison with diffraction results and to provide unit cell snapshots.
Toluene was used in this study as the solvent for TIPS pentacene because of its combination of a high boiling point and solubility. The solution casting of TIPS pentacene was conducted on clean glass slides (received from Fisher Scientific) or precleaned silicon wafers with a $200-\mathrm{nm}$ thermally grown oxide layer inside a 4-in.-diameter Petri dish either with or without the cover. The silicon wafers were cleaned with a seven-step procedure: (1) sonication with deionized water and soap for 10 min, (2) rinsing with copious deionized water, (3) rinsing with copious acetone, (4) boiling in trichloroethylene at $110{ }^{\circ} \mathrm{C}$ for $\sim 10 \mathrm{~min}$, (5) sonication in acetone for $\sim 10 \mathrm{~min},(6)$ boiling in isopropyl alcohol at $82{ }^{\circ} \mathrm{C}$ for $\sim 10 \mathrm{~min}$, and (7) drying with nitrogen gas. For transmission electron microscopy (TEM), solution droplets with a TIPS pentacene concentration of 0.1 wt \% were applied to amorphous carbon thin films (20-50 nm) on top of copper grids (200 or 400 mesh, from Ted Pella) and then dried in air after the extra solution on the grids was wicked from the side with filter paper. Bright-field TEM and electron-diffraction experiments were obtained on a Philips CM12 at $120 \mathrm{kV}$.

Active layers of TIPS pentacene thin-film transistors were fabricated with a solution processing system that could control the temperature and nitrogen gas flushing rate. The flushing rate $(0.04-0.5 \mathrm{~L} / \mathrm{min}$ at room temperature) was monitored and tuned with gas flow meters purchased from Key Instruments. The deposition temperature was controlled by a water bath that surrounded the solution-casting chamber (a glass vial or bottle). The silicon wafers used in this study were heavily arsenide-doped, and the insulator layer was thermally grown silicon dioxide $200 \mathrm{~nm}$ thick $\left(C_{\text {ox }}=17.5 \mathrm{nF} / \mathrm{cm}^{2}\right)$. About 100-nmthick gold electrodes (source and drain) were thermally evaporated through a laser-machined shadow mask. The deposited TIPS pentacene film was about 100-200 nm thick according to AFM measurements, functioning as the active layer of the organic thin-film transistors in a top-contact configuration. The channel width was about 300 $400 \mu \mathrm{m}$, and the channel length was approximately $20-25 \mu \mathrm{m}$ throughout this study. The transistor characteristics were measured with a Keithley 4200 semiconductor parameter analyzer and an Alessi-3200 probe station. Transistor characterization was conducted at room temperature in the ambient atmosphere. Experiments were performed both in the dark and under light. The mobilities were relatively insensitive to light,

Journal of Polymer Science: Part B: Polymer Physics DOI 10.1002/polb 
although transistor off-currents tended to increase with the ambient light intensity. Both transfer $\left(I_{\mathrm{d}}-V_{\mathrm{g}}\right.$, where $I_{\mathrm{d}}$ is the source-drain current and $V_{\mathrm{g}}$ is the gate voltage) and output ( $I_{\mathrm{d}}-$ $V_{\mathrm{d}}$, where $V_{\mathrm{d}}$ is the source-drain voltage) characteristics were tested for each transistor. The $I_{\mathrm{d}}-$ $V_{\mathrm{d}}$ curves were obtained with $V_{\mathrm{g}}$ increasing from -40 to $0 \mathrm{~V}$ and with $V_{\mathrm{d}}$ sweeping from -40 to 0 $\mathrm{V}$. The $I_{\mathrm{d}}-V_{\mathrm{g}}$ curves were collected with $V_{\mathrm{g}}$ increasing from -60 to $20 \mathrm{~V}$ at a constant $V_{\mathrm{d}}$ value of $-40 \mathrm{~V}$. The saturation mobility $(\mu)$ was then extracted from the slope of the transfer curve $V_{\mathrm{g}}-\left(I_{\mathrm{d}}\right)^{1 / 2}$ according to the equation $I_{\mathrm{d}}=$ $\mu W C_{\mathrm{i}}\left(V_{\mathrm{g}}-V_{\mathrm{T}}\right)^{2} / 2 L$, where $W$ and $L$ are the channel width and length, respectively, $C_{\mathrm{i}}$ represents the capacitance in the insulator layer per unit of area, and $V_{\mathrm{T}}$ is the threshold voltage. ${ }^{5}$ Five measurements were taken on the same transistor before and after thermal annealing at $120{ }^{\circ} \mathrm{C}$ for 20 min to study the effect of thermal cracking on charge transport. Most active layers had film coverage above $80 \%$, and the effective mobility was calculated with a normalized channel width (i.e., the width actually covered by the TIPS pentacene film).

Nanoindentation experiments were conducted with a Nano Instruments Nano II and a Berkovitch tip. The loading rates used in this work ranged from 0.05 to $0.8 \mathrm{mN} / \mathrm{s}$.

Surface energy calculations were performed on the Cerius ${ }^{2}$ molecular modeling platform. The size of the simulation system was varied from $45(3 \times 3 \times 5)$ to $405(9 \times 9 \times 5)$ unit cells. Simulation clusters of different sizes were built up with the Non-Periodic Superstructure function of Cerius ${ }^{2}$ Crystal Builder. Each unit cell of TIPS pentacene has two TIPS pentacene molecules $\left(2 \mathrm{C}_{48} \mathrm{H}_{66} \mathrm{Si}_{2}\right)$. The TIPS pentacene model was obtained by single-crystal XRD. The energy minimization of the original structure was performed with the Smart Minimizer option with standard convergence criteria. The simulations for planes with different Miller indices were automated with TCL programming code. Supercells of TIPS pentacene with different sizes (up to 9 times cell parameter $a, 9$ times cell parameter $b$, and 5 times cell parameter $c$ ) were split at certain directions to calculate the surface energy of the corresponding crystalline planes. The two separated halves of the supercells were then pulled toward and then away from each other; meanwhile, the total energy of the system was continuously calculated and output by Cerius $^{2}$ as a function of the separation distance. The out- put text file was processed with the text searching software AquaGrep to obtain the total-energyseparation information.

\section{RESULTS AND DISCUSSION}

\section{Hot-Stage Optical Microscopy}

Characteristic cracking defects developed in the TIPS pentacene thin films upon heating and cooling (Fig. 1). According to differential scanning calorimetry (DSC) and hot-stage optical microscopy, as long as the higher limits of the temperature scans were lower than the combined melting and degradation point (ca. 260$270{ }^{\circ} \mathrm{C}$ ), the TIPS pentacene films could be heated reversibly without losing much of their crystallinity. In Figure 1, several TIPS pentacene single crystals are shown as grown from solution casting (i.e., without any annealing history). The crystals were monitored upon heating to $150{ }^{\circ} \mathrm{C}$ and during the subsequent cooling. There were many reversible fine facets along the (120) planes at room temperature [Fig. 1(d)] that would not appear at higher temperatures [Fig. 1(c)]. ${ }^{6}$ Two major types of cracks were irreversibly formed in the crystals during heating. One of them was parallel, and the other was perpendicular, to the long axes of the individual crystals. On the basis of the electron-diffraction results, ${ }^{7}$ they were the $(\overline{120})$ and (210) types, respectively. From the optical micrographs, it is apparent that the $(\overline{120})$-type cracks were voids resulting from contraction, whereas the (210) ones were overlapping crystals raised after expansion from the two sides. The black rods in Figure 1 show the local orientation of the acene units within the TIPS pentacene molecules, as deduced from the corresponding electron-diffraction patterns. ${ }^{7}$

During heating, the cracks were found to initiate first on the (210) planes (at temperatures of $80-100{ }^{\circ} \mathrm{C}$ ) and then propagate along (120). Longitudinal cracks along (120) were then observed at temperatures about $20^{\circ}$ higher than those of the initial cracking. The initiation temperatures of $(\overline{120})$ cracking were consistently higher than those of (210), whereas the measured expansion along the $(\overline{120})$ planes (3.9 $\pm 1.0 \%$ ) was slightly smaller than the contraction along the $(210)$ planes $(7.5 \pm 1.7 \%){ }^{6}$

After being held for $5 \mathrm{~min}$ at $150{ }^{\circ} \mathrm{C}$ [Fig. $1(\mathrm{c})]$, the films were cooled at $10{ }^{\circ} \mathrm{C} / \mathrm{min}$. Fine 

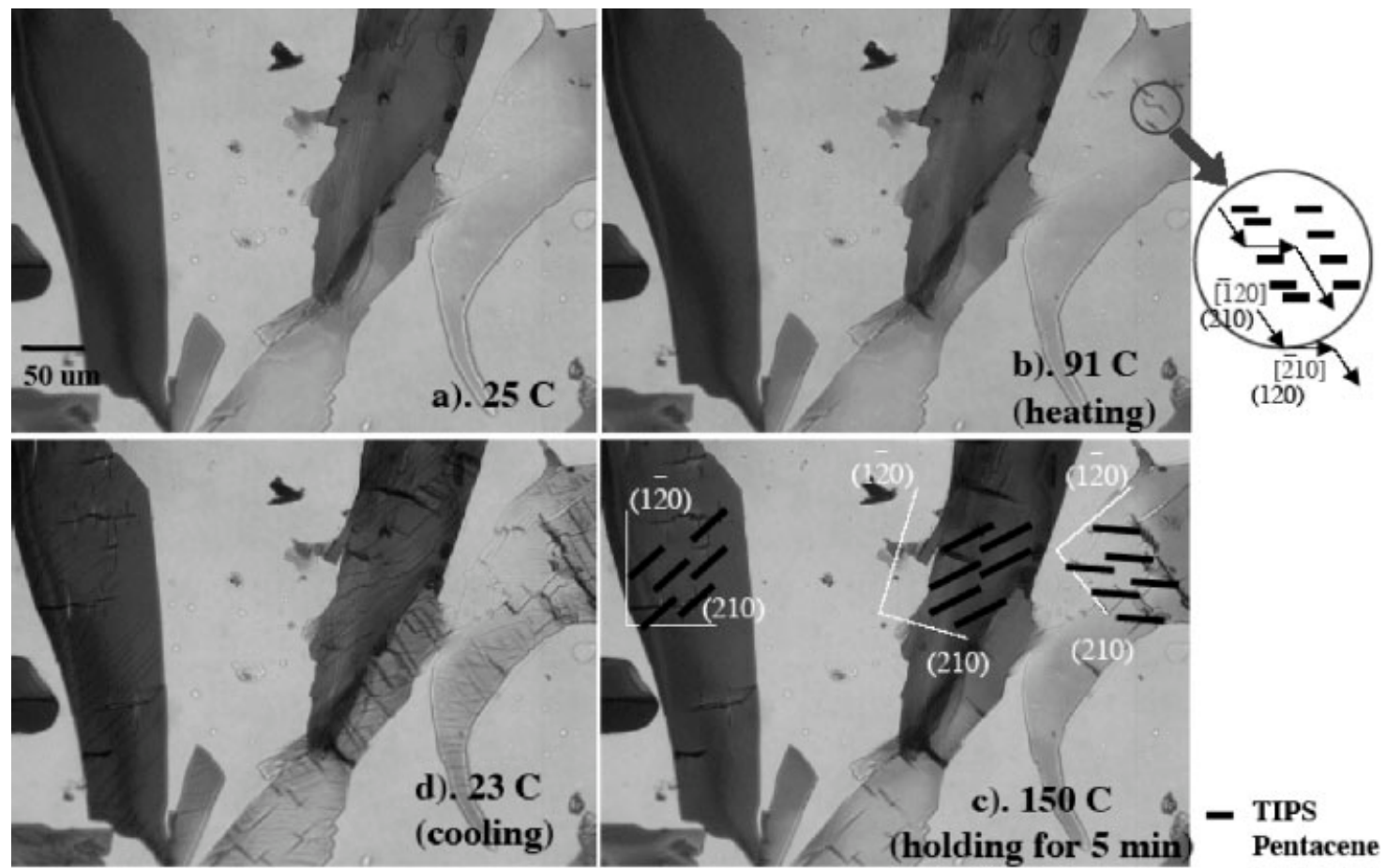

Figure 1. Hot-stage optical images of TIPS pentacene thin films upon heating and cooling from room temperature to $150{ }^{\circ} \mathrm{C}$ : (a) $25{ }^{\circ} \mathrm{C}$ (heating), (b) $91{ }^{\circ} \mathrm{C}$ (heating), (c) $150{ }^{\circ} \mathrm{C}$ (holding for $5 \mathrm{~min}$ ), and (d) $23{ }^{\circ} \mathrm{C}$ (cooling).

facets along (120) started to form during cooling. In addition, many of the fine facets that developed upon cooling initiated along (210) planes and then propagated at (120) again [Fig. 1(d)].

The observed thermal cracking in Figure 1 was associated with a structural phase transition identified with DSC. ${ }^{8}$ Unit cell models for TIPS pentacene crystals both above and below the transition temperature $\left(124{ }^{\circ} \mathrm{C}\right)$ were proposed. At room temperature, we found $a=0.755$ $\mathrm{nm}, b=0.773 \mathrm{~nm}, c=1.676 \mathrm{~nm}, \alpha=89.5, \beta$ $=78.7$, and $\gamma=84.0^{\circ}$, whereas at about $140{ }^{\circ} \mathrm{C}$, the best fit unit cell had $a=0.780 \mathrm{~nm}, b$ $=0.720 \mathrm{~nm}, c=1.720 \mathrm{~nm}, \alpha=90.0, \beta=85.0$, and $\gamma=88.0^{\circ}$. Simulated X-ray and electron diffraction patterns of the two TIPS pentacene crystalline phases were generated and made a reasonable match with the available experimental data. $^{6}$

\section{Mobility Measurements upon Cracking}

Thin-film transistors with solution-processed TIPS pentacene films as active layers were characterized both before and after thermal crack- ing. A top-contact transistor configuration was used, and a top view of a typical TIPS pentacene transistor after thermal cracking is shown in Figure 2(a). The channel length was $20 \mu \mathrm{m}$, and the channel width was $300 \mu \mathrm{m}$. The long axis of the TIPS pentacene films in these transistors ran through the channel roughly parallel, and (210) cracks vertical to the channel direction were found after thermal annealing at $120{ }^{\circ} \mathrm{C}$ for $20 \mathrm{~min}$ in this case. The average grain width of each transistor was determined by the division of the channel width by the number of crystals crossing the channel. Five measurements were made of the grain width to give an estimated standard deviation [Fig. 2(b)].

Depending on the grain width $(5-20 \mu \mathrm{m})$ of the needle-shaped TIPS pentacene crystals, the measured saturation mobility ranged from 0.3 to $1 \mathrm{~cm}^{2} / \mathrm{V}$ s [Fig. 2(b)]. However, after a heat treatment at $120{ }^{\circ} \mathrm{C}$ for $20 \mathrm{~min}$, the mobilities were all reduced to approximately $0.2 \mathrm{~cm}^{2} / \mathrm{V} \mathrm{s}$. In other words, the grain-size dependence of the charge carrier mobility was removed almost completely upon thermal cracking. The mobility reduction upon thermal cracking varied from $50 \%$ in the case of a $5-\mu \mathrm{m}$ grain width to $80 \%$ in

Journal of Polymer Science: Part B: Polymer Physics 

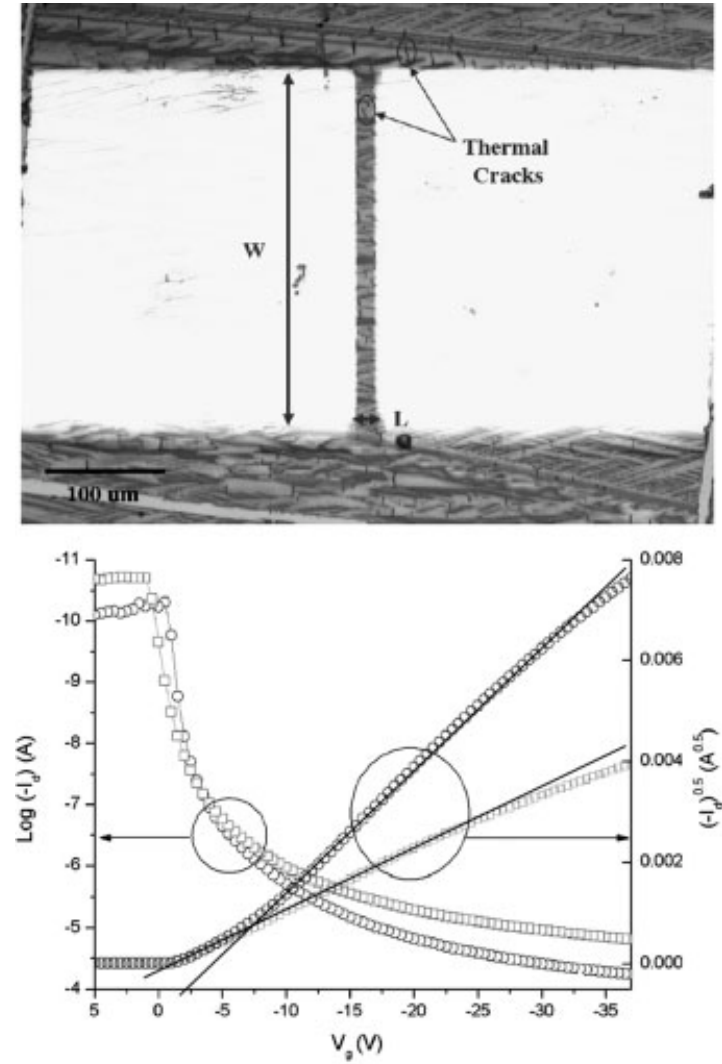

(a)

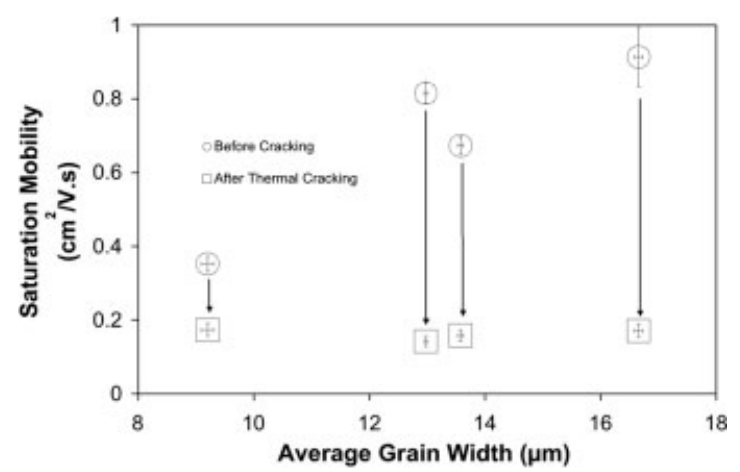

(b)

Figure 2. (a) Effect of (210) cracks on the measured mobilities of TIPS pentacene transistors. The top image shows a top view of a TIPS pentacene thin-film transistor after thermal cracking (width $=20 \mu \mathrm{m}$, length $=300 \mu \mathrm{m})$ with $(210)$ cracks introduced. The bottom image shows transfer characteristics of the same transistor $(\bigcirc)$ before and $(\square)$ after cracking. (b) Effective field-effect mobility measured before and after thermal cracking in four TIPS pentacene transistors with different grain widths. The average grain width was determined by the division of the channel width by the number of crystals crossing the channel. As shown in part a, (210)-type crystal overlapping was responsible for this mobility reduction.

Journal of Polymer Science: Part B: Polymer Physics DOI 10.1002/polb the transistor with the largest grains and highest measured mobility.

Even after substantial cracking, the mobility remained reasonably high. This may be attributed to the fact that the observed (210) cracks involved crystal-crystal overlapping that could still facilitate charge transport. We expect that the (120) cracks that involve void formation would cause a much more dramatic reduction in the mobility if they were introduced vertically to the source-drain direction in the channel area. In addition, with the (210) overlapping that developed upon annealing in the TIPS pentacene thin-film transistors [Fig. 2(a)], it was observed that the mobilities before thermal cracking were heavily grain-size-dependent, whereas the ones after cracking were defect-limited instead and thus exhibited a relatively grain-sizeinsensitive and consistent value.

\section{TEM}

TEM experiments were conducted on TIPS pentacene films after thermal annealing at $150{ }^{\circ} \mathrm{C}$ for $5 \mathrm{~min}$. The crystals were elongated along the $(\overline{120})$ direction, as seen under an optical microscope. Electron diffraction confirmed that the individual crystals were indeed single crystals in nature, with a crystallography that led to characteristic external faceting. In Figure 3, both (120)- and (120)-type cracks are visible within an individual crystal. In Figure 4, the two sets of (120) cracks made it possible for a TIPS pentacene twin boundary to be identified.

\section{Nanoindentation and Mechanical Cracking}

According to optical microscopy, the nanoindentation of solution-cast TIPS pentacene did not generate any obvious macroscopic defects or cracks other than the indentation itself, corresponding to an approximately round-shaped pileup in each case (Fig. 5). A maximum load of $7.5 \mathrm{mN}$ was used to generate the load-displacement information at loading rates ranging from 0.05 to $0.8 \mathrm{mN} / \mathrm{s}$. For each load-displacement curve, a small portion of displacement was recovered upon unloading because of elastic deformation, and the rest of the deformation was plastic and irreversible. From the load-displacement curves, the film hardness was obtained by the division of the load $(P)$ by the indentation area $(A)$ at certain displacement values. $A$ was 


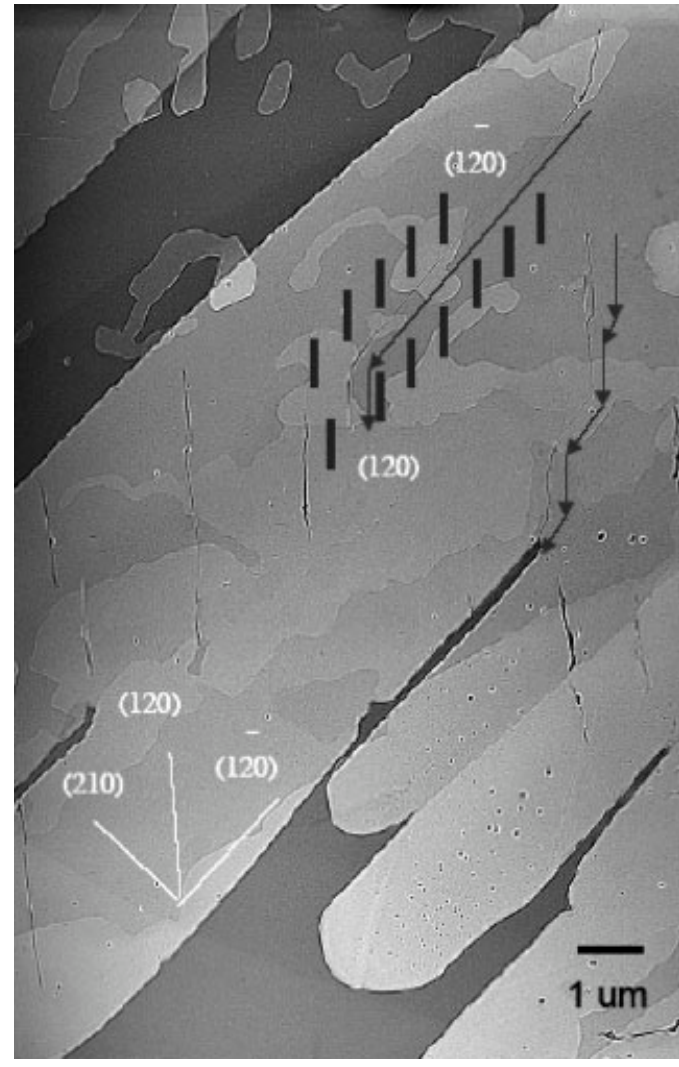

Figure 3. Bright-field TEM image of a TIPS pentacene thin film after thermal annealing at $150{ }^{\circ} \mathrm{C}$ for $5 \mathrm{~min}$. The rods illustrate the local orientation of the acene units within the TIPS pentacene molecules, as deduced from the corresponding electron-diffraction patterns.

calculated on the basis of the indentation tip geometry. At the same time, the film stiffness was given by $d P / d A$, or the changing rate of $P$ upon the variation of $A$, according to the Oliver-Pharr method. ${ }^{9}$

The elastic recovery and loading-rate-dependent stiffness were also seen in the nanoindentation of thermally evaporated pentacene films. ${ }^{10}$ In other words, both of these two organic molecular crystals exhibited characteristics of viscoelasticity. The stiffness of solution-cast TIPS pentacene thin films increased linearly with the loading rate from about $0.05 \mathrm{GPa}$ at $0.05 \mathrm{mN} / \mathrm{s}$ to $0.2 \mathrm{GPa}$ at $0.7 \mathrm{mN} / \mathrm{s}$, whereas the stiffness of thermally evaporated pentacene films went up rapidly at small loading rates, from $0.7 \mathrm{GPa}$ at $0.05 \mathrm{mN} / \mathrm{s}$ to $1.7 \mathrm{GPa}$ at $0.1 \mathrm{mN} / \mathrm{s}$, and then rose more slowly at higher rates to $2.5 \mathrm{GPa}$ at $0.7 \mathrm{mN} / \mathrm{s}$. Although solution-cast TIPS pentacene thin films had much larger grain sizes (generally $>100 \mu \mathrm{m}$ ) than those in thermally evaporated pentacene films (ca. several micrometers), their calculated stiffness values were about an order of magnitude smaller than those of the pentacene films. The alkyl chains in TIPS pentacene evidently leads to a significant drop in the aromatic portion of the unit cell and thus results in a large reduction in the stiffness.

In addition, cracking in TIPS pentacene crystals was studied upon the exertion of an external compressive force. In Figure 6, a TIPS pentacene single crystal was placed between two clean glass slides, and its appearance before and after the application of an external stress of $\sim 70 \mathrm{MPa}$ was imaged. This stress was far beyond the critical cracking point $(\sim 4 \mathrm{MPa})$, and cracks along the crystal long axis were developed all over the crystal edge. By a comparison with the electron-diffraction results, the major cracks in the single crystal upon mechanical deformation were indexed as the $(\overline{120})$ type.

\section{Surface Energy Calculation}

To rationalize the observed thermal and mechanical cracking phenomena, the surface energies of different TIPS pentacene planes were cal-

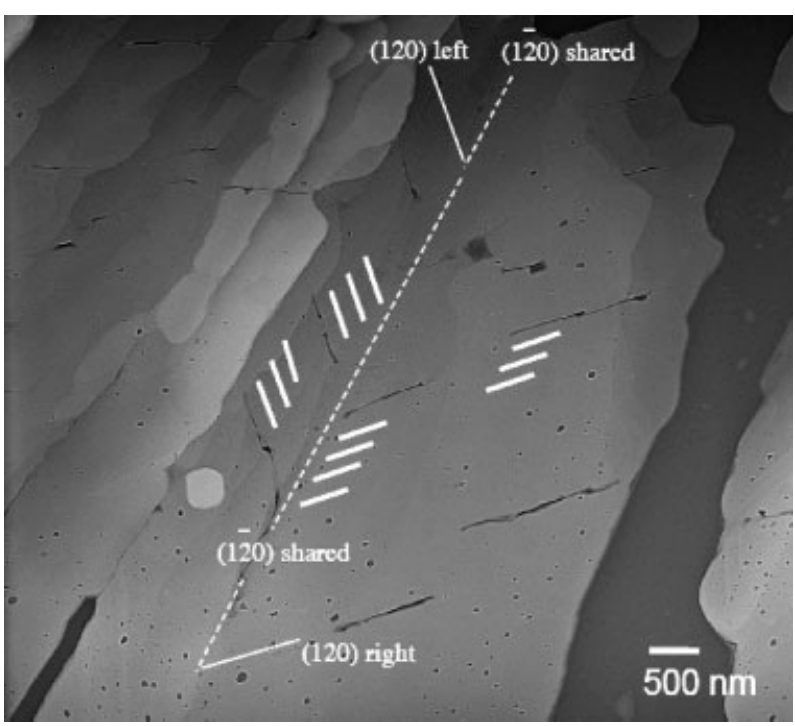

Figure 4. Bright-field TEM image of a TIPS pentacene thin film showing cracks and twin boundaries after the same thermal annealing procedure used for Figure 3. The white rods illustrate the local orientation of the acene units within the TIPS pentacene molecules, as deduced from the corresponding electron-diffraction patterns.

Journal of Polymer Science: Part B: Polymer Physics 

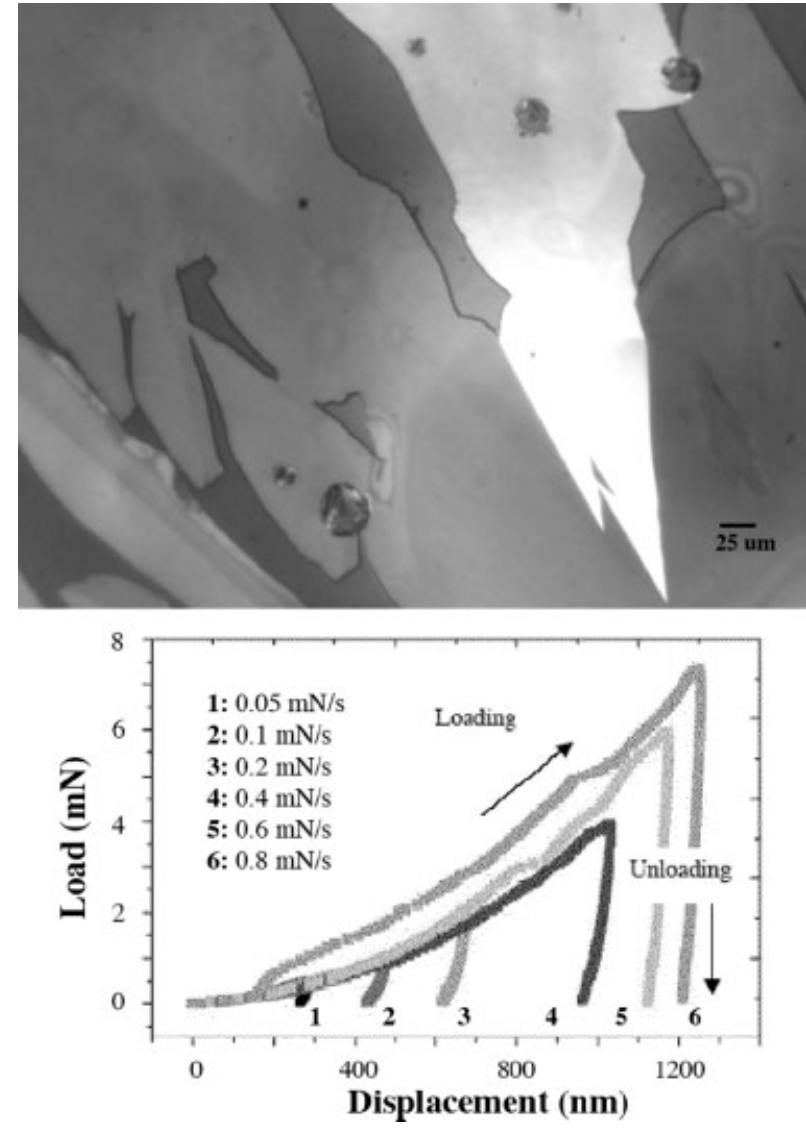

Figure 5. Optical micrograph of TIPS pentacene thin films after nanoindentation (top) and typical load-displacement curves at different loading rates (bottom).

culated on the basis of the energy difference of two crystalline clusters before and after complete separation. The COMPASS force field was used because it was a proven force field that supported molecular simulations of condensed phase materials ${ }^{11}$ and was able to minimize the energy of the TIPS pentacene model without deviating significantly from the experimentally determined unit cell. An ultimate separation of $4 \mathrm{~nm}$ was used, and the total energy upon separation generally stabilized at a distance of $2.5 \mathrm{~nm}$ for most calculations. The energy disparity before and after complete separation was then divided by the newly generated surface area to give values of the corresponding surface energy. In addition, the generation of a specific plane was achieved with proper supercell splitting, and the separation direction was chosen to be normal to the surface plane of interest (Fig. 7).

To verify the validity of this methodology, supercells with different sizes were used, and the surface energies of anthracene and pentacene were calculated and compared with literature values. Similarly to the repeated slab-vacuum geometry with different slab thicknesses and vacuum separation, ${ }^{12-14}$ the cluster size and separation distance were varied systematically in this study to find a convergent value of the surface energy. The calculated surface energy values fluctuated in the beginning and then stabilized as the sizes of the simulation clusters increased. The anthracene (001) surface energy was calculated to be $45 \mathrm{~mJ} / \mathrm{m}^{2}$ with a supercell of $9 a \times 9 b \times 5 c$, which is quite comparable to the reported value of $53 \mathrm{~mJ} / \mathrm{m}^{2}$ with a firstprinciple pseudopotential density-functional calculation. ${ }^{12}$ Surface energies of different planes in the TIPS pentacene crystal lattice were calculated with this methodology (Fig. 8).

Plots of the total energy density versus the reciprocal of the cluster size $(1 / X)$ were used to extend our simulation to a superlattice of infinite size (Fig. 9). The total energy of a given sys-

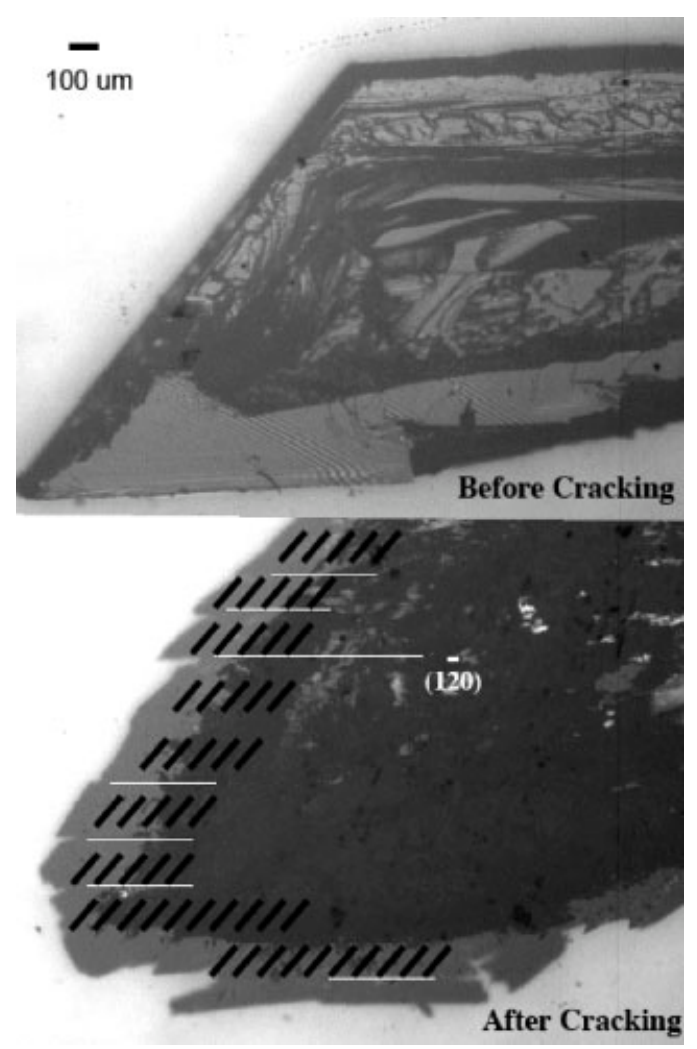

Figure 6. TIPS pentacene single crystal before and after mechanical cracking. The black rods illustrate the local orientation of the acene units within the TIPS pentacene molecules, as deduced from diffraction experiments. 

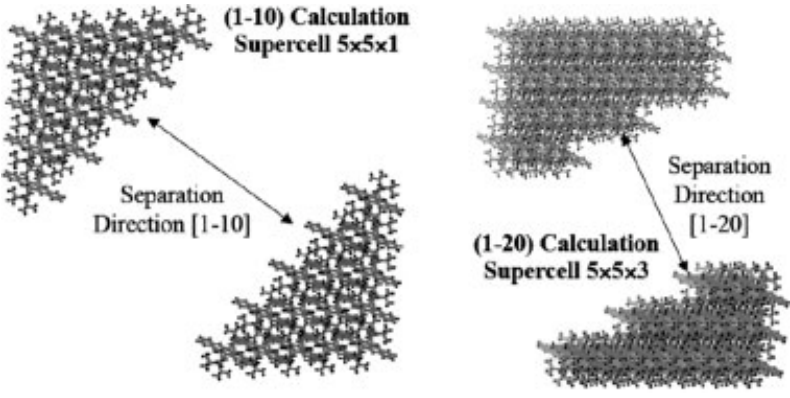

Figure 7. On the left, a $5 \times 5 \times 1$ supercell $(5 a$ in the $x$ dimension, $5 b$ in the $y$ dimension, and $1 c$ in the $z$ dimension) is used for the (110) surface energy calculation. On the right, a $5 \times 5 \times 3$ supercell is used for the $(\overline{120})$ surface energy calculation.

tem is made up of the bulk energy and surface energy:

$$
\begin{gathered}
E_{\text {Total }}=\rho V+\gamma_{(h k l)} A \\
\frac{E_{\text {Total }}}{V}=\rho+\frac{\gamma_{(h k l)} A}{V}
\end{gathered}
$$

where $E_{\text {Total }}$ is the total energy of the system, $\rho$ is the bulk energy density, $\gamma_{(h k l)}$ is the surface energy per unit of area for a certain Millerindex plane $(h k l), A$ is the corresponding surface area, and $V$ is the volume of the supercluster. For the supercell dimensions used here, along the $x$ direction, there are $X$ times of unit cell parameter $a$; along the $y$ direction, there are $X$ times of unit cell parameter $b$; and along the $z$ direction, there are 5 times of unit cell parame-

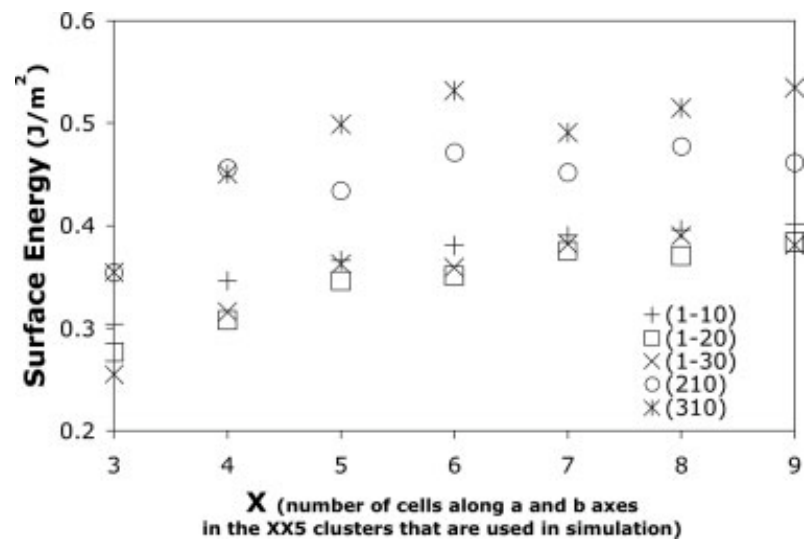

Figure 8. Calculated surface energy for different TIPS pentacene planes with supercell $X X 5$ ( $X$ is the $x$ axis of the plot). According to the calculation, crack planes (210) and ( $\overline{120}$ ) have lower surface energy than planes with similar Miller indices. ter $c$. After substituting in the expressions of $A$ and $V$, we have

$$
\begin{gathered}
A=2 \times(5 c) \times(\alpha X a) \\
V=X \times X \times 5 \times V_{\mathrm{o}} \\
\frac{E_{\text {Total }}}{V}=\rho+\gamma_{(h k l)} \frac{A}{V}=\rho+\left(\frac{2 \gamma_{(h k l)} c \alpha a}{V_{\mathrm{o}}}\right) \frac{1}{X}
\end{gathered}
$$

where $V_{\mathrm{o}}$ is the volume of a unit cell and $\alpha$ is a coefficient that relates cluster size $X$ to the total surface area (which is different for different Miller-index planes). Thus the slope of the total energy density versus $1 / X$ line can be used to extract the corresponding surface energy $\gamma$. The surface energies obtained with this method were close to the ones extracted from Figure 8.

The calculated values for the (110), (120), (130), (210), (310), ( $\overline{110}),(\overline{120})$, and ( $\overline{130})$ planes were all around $300-500 \mathrm{~mJ} / \mathrm{m}^{2}$. The similar values of the surface energies of different planes in TIPS pentacene may explain the multiple cracking directions seen experimentally. In addition, the preferred cracking planes had relatively low surface energies in comparison with other planes with similar Miller indices. For example, the surface energy of $(120)\left(\gamma_{(120)}=336 \mathrm{~mJ} / \mathrm{m}^{2}\right)$ was smaller than those of $(110)\left(\gamma_{(110)}=352 \mathrm{~mJ} / \mathrm{m}^{2}\right)$

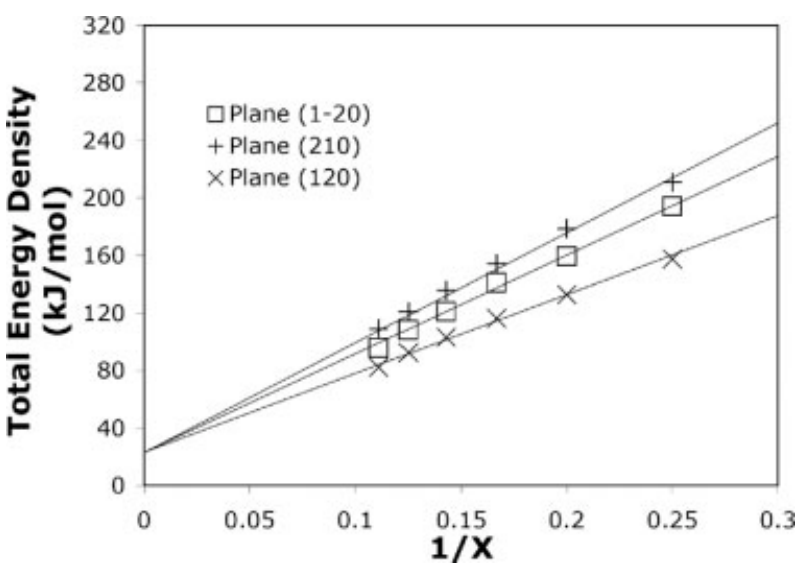

Figure 9. Plots of the total energy density versus the reciprocal of the cluster size $(1 / X) . X$ is the number of cells along axes $a$ and $b$ in the XX5 clusters that are used in the simulation. The simulation results from $X=4-9$ have been extrapolated to yield the total energy density of a superlattice of infinite size. The surface energies of different planes could then be extracted from the slopes of the straight lines.

Journal of Polymer Science: Part B: Polymer Physics 
and $(130)\left(\gamma_{(130)}=352 \mathrm{~mJ} / \mathrm{m}^{2}\right) ; \gamma_{(210)}\left(448 \mathrm{~mJ} / \mathrm{m}^{2}\right)$ was less than $\gamma_{(310)}\left(544 \mathrm{~mJ} / \mathrm{m}^{2}\right)$. Furthermore, the calculated surface energy of the $(\overline{120})$ twin boundaries in Figure 4 was $368 \mathrm{~mJ} / \mathrm{m}^{2}$. This was also lower than the values of other planes in the vicinity, such as $(\overline{110})\left(\gamma_{(\overline{110})}=384 \mathrm{~mJ} / \mathrm{m}^{2}\right)$ and $(\overline{130})\left(\gamma_{(\overline{130})}=400 \mathrm{~mJ} / \mathrm{m}^{2}\right)$.

For cracking planes (210) and (120), the simulation suggested surface energies of 448 and $368 \mathrm{~mJ} / \mathrm{m}^{2}$, which corresponded to 200 and $170 \mathrm{meV} /$ molecule, respectively $\left(16 \mathrm{~mJ} / \mathrm{m}^{2}=1 \mathrm{meV} / \mathrm{A}^{2}\right)$. These numbers, though much lower than the bandgap energy (ca. $1 \mathrm{eV}){ }^{15}$ are quite high when compared with the average thermal energy for a molecule at $100{ }^{\circ} \mathrm{C}\left(32 \mathrm{meV}\right.$, as calculated from $k_{\mathrm{B}} T$, where $k_{\mathrm{B}}$ is the Boltzmann constant and $T$ is the temperature). This means that the thermal energy carried by individual TIPS pentacene molecule alone should not be sufficient to generate those new cracking surfaces. Thus, we believe the internal mechanical pressure built up from the thermally induced phase transition (Fig. 1) provided the energy needed to create the new surfaces along these planes.

The calculated surface energies of TIPS pentacene crystals are significantly larger than the corresponding values for pentacene. For example, $\gamma_{(001)}=76 \mathrm{~mJ} / \mathrm{m}^{2}, \gamma_{(010)}=140 \mathrm{~mJ} / \mathrm{m}^{2}$, and $\gamma_{(110)}=150 \mathrm{~mJ} / \mathrm{m}^{2}$ according to the work of Drummy et al. ${ }^{16}$ on one of the pentacene polymorphs (bulk phase; triclinic: $a=0.628 \mathrm{~nm}, b=$ $0.771 \mathrm{~nm}, c=1.444 \mathrm{~nm}, \alpha=76.75^{\circ}, \beta=88.01^{\circ}$, $\gamma=84.52^{\circ}$ ). For another pentacene polymorph (triclinic: $a=0.79 \mathrm{~nm}, b=0.606 \mathrm{~nm}, c=1.601$ $\mathrm{nm}, \alpha=101.9^{\circ}, \beta=112.6^{\circ}, \gamma=85.8^{\circ}$ ), Northrup et al. ${ }^{12}$ calculated that $\gamma_{(001)}=49.6 \mathrm{~mJ} / \mathrm{m}^{2}, \gamma_{(010)}$ $=102.4 \mathrm{~mJ} / \mathrm{m}^{2}$, and $\gamma_{(110)}=75.2 \mathrm{~mJ} / \mathrm{m}^{2}$. We think the branched shape of the bulky side group largely contributed to the significant differences between the surface energies of TIPS pentacene and pentacene. In addition, surface reorganizations upon the generation of new TIPS pentacene planes were not pursued in this simulation work; therefore, further study is needed to determine the role of this effect.

During the generation of planes $(\overline{120}),(\overline{110})$, and $(\overline{130})$, the total energy upon separation

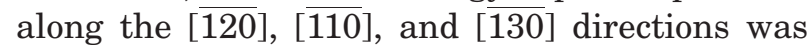
monitored at $0.1-\mathrm{nm}$ intervals with no energy minimization or reconstruction applied (Fig. 10). Before the two halves in a supercell were sepa-
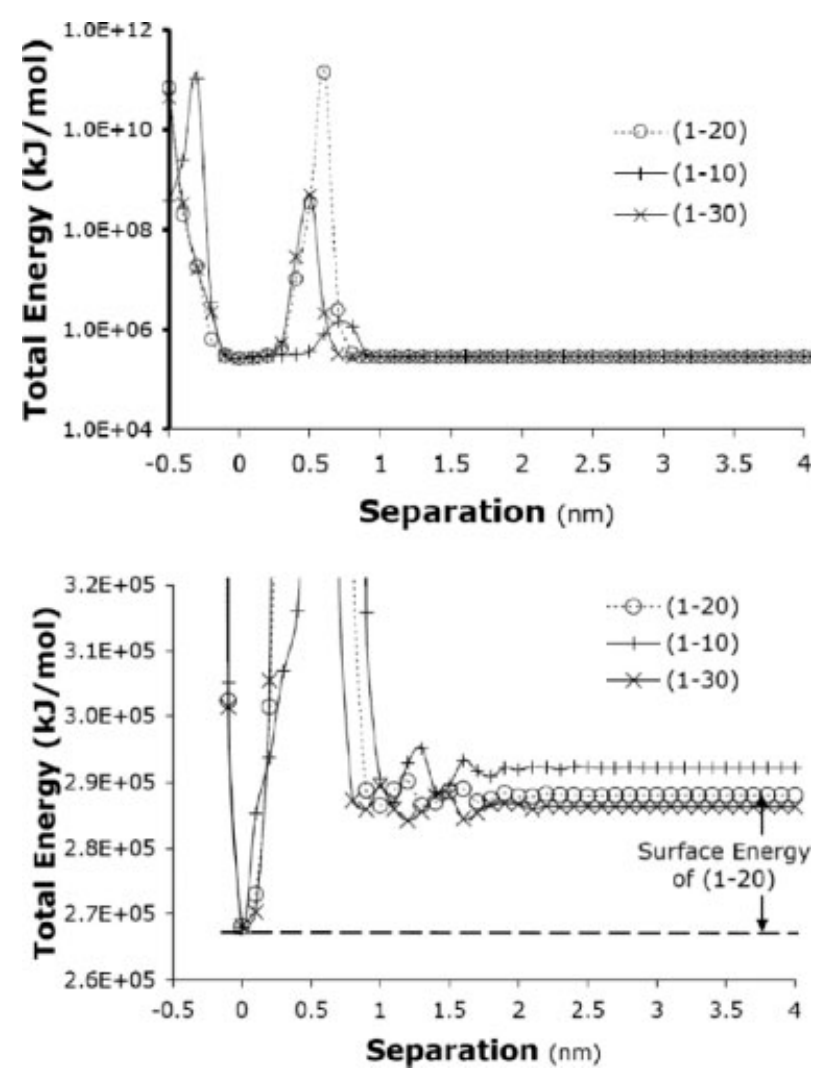

Figure 10. Logarithmic (top) and linear plots (bottom) of total-energy/distance curves during the generation of $(\overline{120}),(\overline{110})$, and $(\overline{130})$ planes upon separation along $[\overline{120}],[\overline{110}]$, and $[\overline{130}]$. A supercluster of $5 a \times 5 b \times 5 c$ was used in this simulation.

rated, they were pushed against each other by $0.5 \mathrm{~nm}$ to give the total energy value at distances closer than equilibrium. This gave rise to a huge peak before the zero separation distance in Figure 10. Because the side groups of TIPS pentacene were interdigitized with each other, when they were pulled apart, the interaction energy became extremely large. Before a steady total energy value was approached at large separations, five or six local minima and maxima were observed, depending on the directions in which the molecules were pulled and on which new planes they eventually formed. This is obviously different from the single-minimum curve of a simple Lennard-Jones potential based on a single repulsion and attractive interaction. The bulky side groups in TIPS pentacene evidently led to this complicated response. This oscillatory behavior in the energy-separation response was also found in pentacene but was much less dra- 


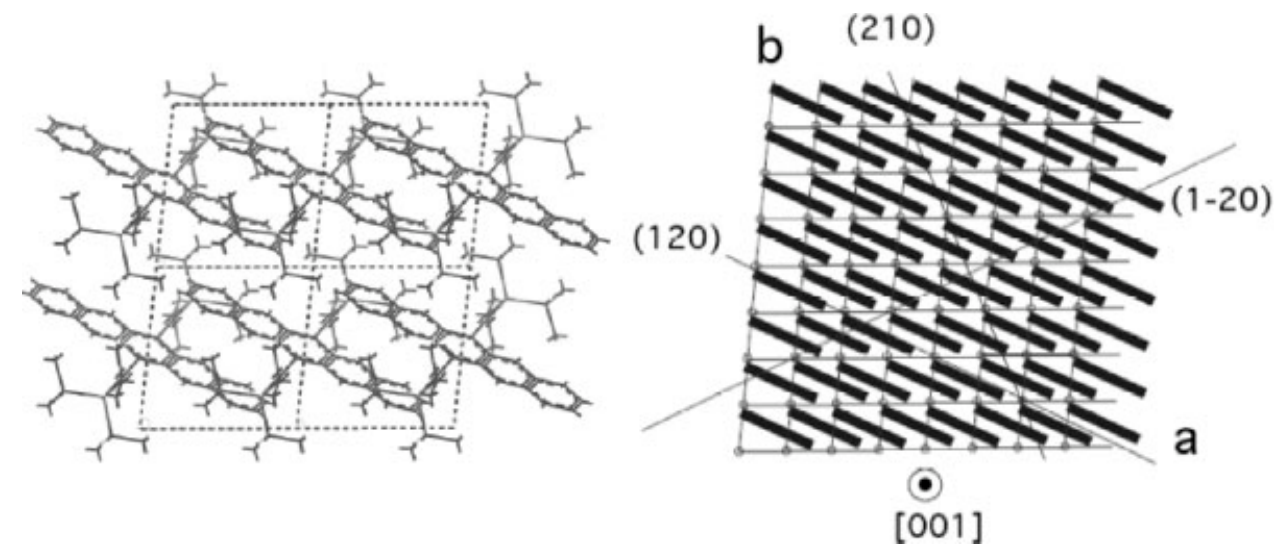

Figure 11. Top view of TIPS pentacene unit cells ([001] projection).

matic. ${ }^{16}$ It is expected that other organic molecular crystals will show similar behavior, especially those with large side-chain substituents. This interdigitation may significantly contribute to the plasticity of TIPS pentacene observed in the nanoindentation experiments.

During thermal cracking, the (210) planes had a lower cracking initiation temperature than the $(\overline{120})$ planes. Our surface energy calculations suggested that $\gamma_{(210)}\left(448 \mathrm{~mJ} / \mathrm{m}^{2}\right)$ was actually higher than $\gamma_{(\overline{120})}\left(368 \mathrm{~mJ} / \mathrm{m}^{2}\right)$. Therefore, the reason for the earlier crack initiation of (210) planes was most likely kinetic pathway effects rather than thermodynamic driving forces. As one can see from the unit cell of TIPS pentacene (Fig. 11), to generate new $(\overline{120})$ planes, the bulky TIPS side groups will interfere with one another during separation, whereas the energy barriers encountered during breaking along the (210) planes will be much smaller. Therefore, although they have relatively lower surface energy overall, the $(\overline{120})$ planes require larger thermal energies (higher temperatures) than the (210) planes to form the cracks in the first place.

\section{CONCLUSIONS}

In this work, characteristic thermal and mechanical crack formation along the (210), (120), and $(\overline{120})$ planes in TIPS pentacene was examined in terms of its crystallography, surface energy, and important impact on charge transport. Interestingly, the mobility of TIPS pentacene thin-film transistors was found to consistently drop to a non-zero small value $\left(0.2 \mathrm{~cm}^{2} / \mathrm{V}\right.$ s) after thermal cracking because of the moun- tains that developed along (210) on account of thermal expansion along the film's long axis.

The authors are grateful to the Office of Naval Research and the National Science Foundation for its financial support (DMR-0084304 and DMR-6518079). Hot-stage X-ray diffraction experiments were performed in Omar M. Yaghi's laboratory in the Department of Chemistry at the University of Michigan at Ann Arbor. Technical staff Ying Qi of the Department of Materials Science and Engineering at the University of Michigan helped greatly in maintaining the hot-stage light microscope, X-ray diffraction system, and differential scanning calorimetry equipment. Transmission electron microscopy studies were conducted at the Electron Microbeam Analysis Laboratory at the University of Michigan at Ann Arbor with the great assistance of the laboratory managers: John Mansfield, Kai Sun, and Carl Henderson. J. Chen appreciates constructive discussions with Yanbin Chen of the Department of Materials Science and Engineering at the University of Michigan.

\section{REFERENCES AND NOTES}

1. Nelson, S. F.; Lin, Y.-Y.; Gundlach, D. J.; Jackson, T. N. Appl Phys Lett 1998, 72, 1854-1856.

2. Shtein, M.; Mapel, J.; Benziger, J. B.; Forrest, S. R. Appl Phys Lett 2002, 81, 268-270.

3. Anthony, J. E.; Brooks, J. S.; Eaton, D. L.; Parkin S. R. J Am Chem Soc 2001, 123, 9482 9483.

4. Anthony, J. E.; Eaton, D. L.; Parkin, S. R. Org Lett 2002, 4, 15-18.

5. Dimitrakopoulos, C. D.; Malenfant, P. R. L. Adv Mater 2002, 14, 99-117.

6. Chen, J.; Anthony, J. E.; Martin, D. C. J Phys Chem B 2006, 110, 16397-16403.

7. Chen, J. Structure, processing and properties of the organic molecular semiconductor triisopropyl-

Journal of Polymer Science: Part B: Polymer Physics DOI 10.1002/polb 
silylethnyl (TIPS) pentacene, Ph.D. dissertation, University of Michigan, 2006.

8. Chen, J.; Anthony, J. E.; Martin, D. C. Polym Prepr 2005, 46, 539-540.

9. Pharr, G. M. Mater Sci Eng A 1998, 253, 151-159.

10. Drummy, L. F.; Miska, P. K.; Martin, D. C. J Mater Sci 2004, 39, 1-10.

11. Sun, H. J Phys Chem B 1998, 102, 73387364 .

12. Northrup, J. E.; Tiago, M. L.; Louie, S. G. Phys Rev B 2002, 66, 121404.
13. Oviedo, J.; Gillan, M. J. Surf Sci 2000, 463, 93101.

14. Bates, S. P.; Kresse, G.; Gillan, M. J. Surf Sci 1997, 385, 386-394.

15. Haddon, R. C.; Chi, X.; Itkis, M. E.; Anthony, J. E.; Eaton, D. L.; Siegrist, T.; Mattheus, C. C.; Palstra, T. T. M. J Phys Chem B 2002, 106, 82888292.

16. Drummy, L. F.; Miska, P. K.; Alberts, D.; Lee, N.; Martin, D. C. J Phys Chem B 2006, 110, 60666071. 